\title{
ENDOSCOPIC TREATMENT FOR UPPER GI BLEEDING. REVALIDATION OF FORREST CLASSIFICATION
}

\author{
Ahed EL-KHATIB, MD, Catalin Alius, MD, Lecturer Dragos Serban, MD, PhD \\ University Hospital, Bucharest
}

\begin{abstract}
Despite rapid advances in endoscopic treatments and the development of updated and evidence based guidelines, morbidity and mortality in upper gastrointestinal bleeding (UGIB) remains high. A recent study conducted in a tertiary centre on 80 consecutive patients with UGIB demonstrated that lower doses of Adrenaline 1:10000 and diathermy were superior to high doses of Adrenaline injections only, but failed to reduce the mortality rates, despite a reduction in the need for transfusion, second look and surgical interventions. We believe that comorbid conditions have greater influence on survival rates in patients with UGIB than the achievement of immediate haemostasis and we attribute a paramount importance to rapid and correct resuscitation of the bleeding patient prior to any endoscopic assessment.
\end{abstract}

Keywords: Upper GI bleeding, endoscopic treatment, Forrest classification

\section{INTRODUCTION}

Upper gastrointestinal bleeding (UGIB) is becoming a growing problem at the emergency departments (ED) with the aging of the population, increased number and severity of comorbidities in patients and raise in the use of ulcerogenic medication (1-3).

We define upper GI bleeding as any source of bleeding arising from above the ligament of Treitz. This type of bleeding could become clinically apparent through melena, haematemesis or PR (per rectum) bleeding and clinical instability.

The Forrest classification offers an excellent tool for predicting the probability of rebleeding in UGIB $(3,5)$ guiding the endoscopist in deciding which lesions require endoscopic therapy $(4,5)$. If correlated with clinical scoring systems it facilitates improvement of patients outcomes (6).

Endoscopic therapy should be offered only to actively bleeding lesions (spurting vessel, oozing hemorrhage) or high risk lesions with stigmata of recent hemorrhage (visible non bleeding vessel and adherent clots - when technically possible) (7).

Multimodal endoscopic therapy has been shown to be superior to single methods of achieving hae- mostasis (7). The newest guidelines recommend the association of two endoscopic modalities in order to reduce the rebleeding episodes, yet in some centers single modalities are still employed for various reasons with variable results.

We conducted a study in our ED on 80 consecutive patients with UGIB and compared the efficacy of low dose Adrenaline and diathermy versus high doses of Adrenaline 1:10,000. The patients receiving higher doses of Adrenaline had inferior control of the bleeding but the overall mortality has not changed.

Multimodal endoscopic therapy was superior to injection only but it did not improve mortality probably because of independent factors like the co-morbid conditions of the patients.

Both multimodal endoscopic therapy and high doses of Adrenaline 1:10,000 injections correlated well with a reduction of the transfusions, second look endoscopies and surgical interventions.

We believe that with the increasing age of the population the cumulus of co-morbid conditions has a greater impact on the mortality than the achievement of rapid haemostasis in UGIB. 


\section{PATIENTS AND METHODS}

We conducted a retrospective study on 80 patients with UGIB who had emergency endoscopies in an ED department at a tertiary centre. They all presented as acute patients with clinical suspicion of UGIB or had been diagnosed during the initial assessment with UGIB.

Charts of all the 80 patients were analyzed for history details, clinical and endoscopic variables.

We calculated an initial GBS score and a Rockall score based on the data available at the time of presentation and we categorized the endoscopic findings according to the Forrest classification. None of the patients were suitable candidates for follow up endoscopy in the community therefore they received endoscopic evaluation as soon as possible.

The candidates for endoscopic treatment received low or high doses of Adrenaline 1:10,000 and, if in the low Adrenaline dose arm diathermy was employed to secure the result. Rebleeding patients had a second look and another endoscopic attempt to achieve haemostasis before they were offered surgery. This small cohort of patients had injections, diathermy and clips depending on the endoscopic findings.

The Forrest classification was developed to predict mortality in UGIB patients but it is very useful in predicting rebleeding.

All lesion showing acute bleeding (spurting hemorrhage, oozing haemorrhage) and stigmata of recent bleeding (non-bleeding but visible vessel and lesions with adherent clots) were offered endoscopic treatment. Patients in whom adherent clots could not be mobilized with a vigorous wash were not offered endoscopic treatment unless they showed signs of clinical deterioration suggesting rebleeding.
TABLE 1. Forrest Classification for bleeding peptic lesions

\begin{tabular}{|l|l|l|}
\hline I. Active Bleeding & $\begin{array}{l}\text { II. Stigmata of recent } \\
\text { haemorrage }\end{array}$ & $\begin{array}{l}\text { III. Lesions } \\
\text { without signs } \\
\text { of recent } \\
\text { haemorrhage }\end{array}$ \\
\hline $\begin{array}{l}\text { la: Spurting } \\
\text { haemorrhage }\end{array}$ & Ila: Vissible vessel & \\
\cline { 1 - 1 } $\begin{array}{l}\text { Ib: Oozing } \\
\text { haemorrhage }\end{array}$ & Ilb: Adherent clot & \\
\hline & Ilc: Hematin covered lesion & \\
\hline
\end{tabular}

This classification is a reliable predictor for rebleeding, but does not predict mortality as well as other scores which also incorporates clinical variables. For this reason the use of Rockall and the Glasgow-Blanchard Score (GBS) is complementary to the endoscopic assessment in order to stratify the patients correctly (8-10).

The evaluation protocol made use of three endoscopic scoring systems, namely the Forrest classification for describing the lesions, the GBS score and the Rockall score for assessing the biological status of the patient at presentation. The GBS score incorporates blood results and can be useful in detecting acute anemia, high levels of urea as a consequence of dehydration or increased absorption from the gut and early signs of renal failure. The Rockall score integrates endoscopic findings with clinical variables and takes into account the comorbid conditions of the patients in order to predict the risk of death. Although age is not the best predictor of mortality if analyzed separately in an aging population, the correlations made with criteria for analyzing hipovolaemic shock and endoscopic diagnoses make this score a reliable tool in identifying patients at risk for poor outcomes.

All our patients were offered endoscopy within few hours since they presented to ED because none had a Rockall or a GBS as low as to allow them to be treated in the community. The GBS was classically associated with a good prediction of the need

TABLE 2. The Rockal score

\begin{tabular}{|c|c|c|c|c|}
\hline & 0 & 1 & 2 & 3 \\
\hline Age & $<60$ & $60-79$ & $>80$ & \\
\hline Shock & $\begin{array}{l}\text { Pulse }<100 \\
\text { BP }>100\end{array}$ & $\begin{array}{c}\text { Pulse }>100 \\
\text { BP }<100\end{array}$ & $\begin{array}{c}\text { Pulse }<100 \\
\mathrm{BP}<100 \\
\end{array}$ & \\
\hline Comorbidities & None & & $\begin{array}{l}\text { Heart failure/Ischemic } \\
\text { heart disease }\end{array}$ & $\begin{array}{l}\text { Renal failure } \\
\text { Liver failure } \\
\text { Disseminated } \\
\text { malignancy }\end{array}$ \\
\hline $\begin{array}{l}\text { Endocopic } \\
\text { signs of } \\
\text { bleeding }\end{array}$ & None/dark spots & & $\begin{array}{c}\text { Blood/adherent clot/ } \\
\text { Visible or spurting } \\
\text { vessel }\end{array}$ & \\
\hline Diagnosis & $\begin{array}{l}\text { Mallory Weiss/no } \\
\text { finding }\end{array}$ & $\begin{array}{l}\text { All other } \\
\text { diagnoses }\end{array}$ & $\begin{array}{c}\text { Upper GI } \\
\text { malignancies }\end{array}$ & \\
\hline
\end{tabular}


for endoscopic treatment, but it could be used to predict the need for transfusions, surgical interventions and up to a point, mortality (10).

We used SPSS for data processing and Excel. The study aimed to compare low doses of Adrenaline and diathermy versus high doses of Adrenaline injections used for achievement of haemostasis. Secondary objectives where to determine if low doses of adrenaline prevented precipitation of cardiovascular events in the anemic patient and if there was any improvement in the mortality rates with the use of scoring systems.

\section{RESULTS}

TABLE 3. The GBS score

\begin{tabular}{|c|c|}
\hline $\begin{array}{l}\text { Blood urea (mmol/L) } \\
\cdot 6.5-7.9=2 \text { points } \\
\cdot 8.0-9.9=3 \text { points } \\
\cdot 10.0-25.0=4 \text { points } \\
\cdot>25.0=6 \text { points }\end{array}$ & $\begin{array}{l}\cdot \\
\cdot 3 \\
\cdot 3 \\
\cdot 4\end{array}$ \\
\hline $\begin{array}{l}\text { Haemoglobin for men }(\mathrm{g} / \mathrm{L}) \\
\cdot 120-129=1 \text { points } \\
\cdot 100-119=3 \text { points } \\
\cdot<100=6 \text { points }\end{array}$ & $\begin{array}{l}\cdot 1 \\
\cdot 3 \\
\cdot 6\end{array}$ \\
\hline $\begin{array}{l}\text { Haemoglobin for women (g/L) } \\
\cdot 100-119=1 \text { point } \\
\cdot<100=6 \text { point }\end{array}$ & $\begin{array}{l}\cdot 1 \\
\cdot 6\end{array}$ \\
\hline $\begin{array}{l}\text { Systolic blood pressure }(\mathrm{mmHg}) \\
\text { - } 100-109=1 \text { point } \\
\cdot 90-99=2 \text { point } \\
\cdot<90=3 \text { point }\end{array}$ & $\begin{array}{l}\cdot 1 \\
\cdot 2 \\
\cdot 3\end{array}$ \\
\hline $\begin{array}{l}\text { Other markers Pulse } \\
\text { - }>=100 / \text { min }=1 \text { point } \\
\text { - presentation with melaena }=1 \text { point } \\
\text { - } \text { presentation with syncope }=2 \text { point } \\
\text { - hepatic disease* }=2 \text { point } \\
\text { - } \text { cardiac failure } * *=2 \text { point }^{*}\end{array}$ & $\begin{array}{l}\cdot 1 \\
\cdot 1 \\
\cdot 1 \\
\cdot 2 \\
\cdot 2\end{array}$ \\
\hline
\end{tabular}

We analyzed 80 consecutive patients with UGIB requiring urgent endoscopy in the ED department.

A proportion of $26 \%$ of patients were female patients and $74 \%$ male.

We concluded that the number of patients requiring endoscopic treatment was $84 \%$.Out of these only 15 patients rebled and only 3 required surgery.

The findings at the endoscopy placed $21.8 \%$ in the active bleeding category, $63.3 \%$ in the stigmata of recent bleeding category and only $14.6 \%$ in the third category.

The largest proportion of rebleeds were attributed to the second category, namely the IIa and IIb lesions.

A number of $34 \%$ of patients required small doses of Adrenaline to control the bleeding and $66 \%$ needed larger doses.
In patients requiring small doses of Adrenaline to control the bleeding another endoscopic modality had been used to prevent rebleeding.

We did not find any correlation between the amount of Adrenaline used and precipitation of cardiovascular events nor it influenced the overall mortality, need for transfusion and the length of stay in the hospital.

TABLE 4. Stratification of patients according to the Forrest classification

\begin{tabular}{|l|c|c|c|}
\hline $\begin{array}{l}\text { Endoscopic } \\
\text { diagnosis }\end{array}$ & $\begin{array}{c}\text { Number of } \\
\text { patients }\end{array}$ & $\begin{array}{c}\text { Number of } \\
\text { rebleeding }\end{array}$ & $\begin{array}{c}\text { Percentage of } \\
\text { rebleeding }\end{array}$ \\
\hline Forrest la & $3(3.65 \%)$ & 0 & $0 \%$ \\
\hline Forrest Ib & $15(18.2 \%)$ & 3 & $20 \%$ \\
\hline Forrest Ila & $28(34.1 \%)$ & 5 & $17 \%$ \\
\hline Forrest IIb & $24(29.2 \%)$ & 7 & $29 \%$ \\
\hline Forrest Ilc.III & $12(14.6 \%)$ & 0 & $0 \%$ \\
\hline
\end{tabular}

\section{DISCUSSIONS AND CONCLUSIONS}

This study had been envisaged having in mind that higher doses of vasopressor medication could precipitate cardiovascular events in elderly patients presenting with UGIB.

The biggest predictor of death in the cohort we analyzed was not the severity of the lesion or the rebleeding but the associated co-morbidities of the patients. We believe that in order to reduce the mortality related to UGIB the initial resuscitation has to be conducted rigorously, with minimal delay and supported by evidence based developed guidelines.

The aging of the population and the increase of the ulcerogenic medicines have a big impact on the outcome in UGIB patients through the cumulative effect of the co-morbid conditions.

Through our study we revalidated the predictive value of the Forrest classification for rebleeding. Once the bleeding had been stopped by Adrenaline injection 1:10,000 we did not employ a second haemostatic modality in the higher dose Adrenaline arm, but in order to prevent rebleeding we used diathermy in the low doses arm. The use of lower doses of vasoactive medication is a useful point of view especially in the anemic elderly patient in whom high doses of vasopressors could lead to precipitation of cardiovascular events.

In our study the percentage of male patients was very high although we enrolled consecutive patients in the study. We believe that the discrepancies are related to the demographic particularities

The immense value of scoring systems in the management of UGIB patients has been reconfirmed. These rapid and reproducible clinical as- 
sessment tools are of immense value in recognizing the patients at risk and should prompt immediate action. In the critically ill patient early recognition of the severity of the illness is crucial for a better outcome.

We conducted a small study on 80 consecutive patients presenting with signs of UGIB at the ED and requiring endoscopy. The Forrest classification as a predictor for rebleeding in patients with grade I and II lesions has been reconfirmed. We believe that spreading of multimodal endoscopic treatment could improve outcomes and prevent precipitation of cardiovascular events in the anemic patient who requires vasopresos for bleeding lesions. A larger study has to be designed in order to validate these findings.

\section{REFERENCES}

1. Lanas A., Garcia-Rodriguez L.A., Polo-Tomas M. Time trends and impact of upper and lower gastrointestinal bleeding and perforation in clinical practice. Am J Gastroenterol 2009; 104:1633-1641.

2. Crooks C., Card T. Reductions in 28-day mortality following hospital admission for upper gastrointestinal hemorrhage. Gastroenterology 2011; 141:62-70.

3. Parker D.R., Luo X., Jalbert J., et al. Impact of upper and lower gastrointestinal blood loss on healthcare utilization and costs: a systematic review. J Med Econ 2011; 14:279-87.

4. Lau J.Y., et al. Systematic review of the epidemiology of complicated peptic ulcer disease: incidence, recurrence, risk factors and mortality. Digestion 2011; 84:102-113.

5. Akande O.A., Ebenezer A.A., Taiwo H.R., et al. Application of Forrest Classifiction in the Risk Assessment and Prediction of Reb leeding in Patients with Bleeding Peptic Ulcer in Ado-Ekiti, Nigeria. American Journal of Medicine and Medical Sciences 2014; 4(4):108-113

6. Hadzibulic E., Govedarica S. Significance of Forrest Classification, Rockall's and Blatchford's risk scoring system in prediction of rebleeding in peptic ulcer disease. Acta Medica Medianae 2007; 46:38-43

7. Scottish Intercollegiate Guidelines Network, management of acute upper and lower GI bleeding, Edinburgh, 2010.

8. Monteiro S., Goncalves T.C. Magalhaes Joana, et al. Upper gastrointestinal bleeding risk scores: Who, when and why?.World J Gastrointest Pathophysiol 2016; 7(1):86-96

9. Chen I.C., Hung M.S., Chiu T.F., et al. Risk scoring systems to predict need for clinical intervention for patients with nonvariceal upper gastrointestinal tract bleeding. Am J Emerg Med 2007; 25: 774-779

10. Attar A., Sebbagh V., Vicaut E., et al. Urgent endoscopy in severe non-variceal upper gastrointestinal hemorrhage: does the GlasgowBlatchford score help endoscopists?. Scand J Gastroenterol 2012; 47: 1086-1093

11. Wong S.K.H., Yu L.M., Lau J.Y.M., et al. Prediction of therapeutic failure after adrenaline injection plus heaterprobe treatment in patients with bleeding peptic ulcer. Gut 2002; 50:322-5.

12. Brullet E., Calvet X., Campo R., et al. Factors predicting failure of endoscopic injection therapy in bleeding duodenal ulcer. Gastrointest Endosc 1996; 43:111-6.

13. Holster I.L., Kuipers E.J. Update on the endoscopic management of peptic ulcer bleeding. Curr Gastroenterol Rep 2011; 13: 525-531

14. Laine L., Jensen D.M. Management of patients with ulcer bleeding. Am J Gastroenterol 2012; 107: 345-360

15. Loftus I. et al. Care of the critically ill patient. $3^{\text {rd }}$ edition. New York: CRC Press Taylor and Francis Group, 2010 